EGU21-12357, updated on 10 Mar 2021

https://doi.org/10.5194/egusphere-egu21-12357

EGU General Assembly 2021

(c) Author(s) 2021. This work is distributed under

the Creative Commons Attribution 4.0 License.

\title{
Target food security: assimilating ultra-high resolution satellite images into a crop-yield forecasting model
}

\author{
Matteo G. Ziliani ${ }^{1}$, Bruno Aragon ${ }^{1}$, Trenton Franz ${ }^{2}$, Ibrahim Hoteit ${ }^{1}$, Justin Sheffield ${ }^{3}$, and Matthew \\ F. McCabe ${ }^{1}$ \\ ${ }^{1}$ KAUST, Thuwal, Saudi Arabia \\ ${ }^{2}$ University of Nebraska-Lincoln, Lincoln, USA \\ ${ }^{3}$ University of Southampton, Southampton, United Kingdom
}

Assimilating biophysical metrics from remote sensing platforms into crop-yield forecasting models can increase overall model performance. Recent advances in remote sensing technologies provide an unprecedented resource for Earth observation that has both, spatial and temporal resolutions appropriate for precision agriculture applications. Furthermore, computationally efficient assimilation techniques can integrate these new satellite-derived products into modeling frameworks. To date, such modeling approaches work at the regional scale, with comparatively few studies examining the integration of remote sensing and crop-yield modeling at intra-field resolutions. In this study, we investigate the potential of assimilating daily, $3 \mathrm{~m}$ satellite-derived leaf area index (LAI) into the Agricultural Production Systems sIMulator (APSIM) for crop yield estimation in a rainfed corn field located in Nebraska. The impact of the number of satellite images and the definition of homogeneous spatial units required to re-initialize input parameters was also evaluated. Results show that the observed spatial variability of LAI within the maize field can effectively drive the crop simulation model and enhance yield forecasting that takes into account intra-field variability. The detection of intra-field biophysical metrics is particularly valuable since it may be employed to infer inefficiency problems at different stages of the season, and hence drive specific and localized management decisions for improving the final crop yield. 